\title{
A Novel Approach to Joint User Selection and Precoding for Multiuser MISO Downlink Channels
}

\author{
Ashok Bandi, Bhavani Shankar Mysore R , Sina Maleki, Symeon Chatzinotas and Björn Ottersten \\ Interdisciplinary Centre for Security, Reliability and Trust (SnT), the University of Luxembourg, Luxembourg. \\ Email: \{ashok.bandi, bhavani.shankar, sina.maleki, symeon.chatzinotas, bjorn.ottersten\}@uni.lu
}

\begin{abstract}
The downlink capacity of a unicast network with a large number of users than the base station transmit antennas depends on user selection and interference among the selected users. Various suboptimal selection schemes in combination with suboptimal or optimal precoding have been proposed in the literature, and some of these techniques asymptotically achieve the sum capacity of DPC, as the number of users goes to infinity. In the previous works, the joint design problem is addressed as a decoupled problem of selection and precoding either at the design level or the solution level. In this work, we focus on the design of a joint solution to the joint design problem, which has the potential to achieve better sum capacity than the aforementioned designs. First, we develop a framework to formulate the joint design problem, with the objective of maximizing Weighted sum rate (WSR). Further, we reformulate the resulting NP-hard WSR maximization problem as a difference-of-concave (DC) functions with novel rate reformulations. Finally, the DC maximization problem is solved using the convex-concave procedure, which is guaranteed to converge to a stationary point. Through simulations, we show the efficacy of proposed solution over the stateof-the-art designs.
\end{abstract}

Index Terms-User selection, Precoding, Difference-of-convex (DC) problem, convex-concave procedure (CCP)

\section{INTRODUCTION}

With the adaptation of full frequency reuse in the next generation cellular networks, interference among the simultaneously served users becomes a fundamental limiting factor thwarting the achievement of network capacity. DPC achieves capacity but is rather complex. Hence, low complexity linear precoding schemes have gain popularity in literature [1]-[3]. Further, in a network with a large number of users compared to BS transmit antennas, selection of users for simultaneous transmission, in addition to precoding, is essential to improve the network capacity [4], [5].

In this work, we address the joint design of user selection and precoding, which we simply refer to as joint design, with the objective of maximizing weighted sum rate (WSR) subject to the classical total transmit power constraint over the downlink. Due to non-convexity of WSR (see in [6], [7]) and combinatorial nature of user selection problem, the optimal solution requires an exhaustive search over two coupled spaces namely user selection (Boolean space) and precoding (complex continuous space). Exhaustive search based algorithms, in general, have exponential complexity prohibiting their use for practical realizable system dimensions. A low complexity

This work is supported in part by luxembourgish national fund FNR projects PROSAT and SATSENT. decoupling of the selection and precoding at the design level is considered for single cell networks in [4], [5] and for multicell networks [8]-[13]. In [4] and [5], authors propose heuristic suboptimal user selection schemes followed by zero-forcing precoding which asymptotically achieves sum capacity of DPC in single cell networks. However, the heuristic condition of selecting the users with orthogonal channels is only optimum for a larger pool size of users compared to the selected users.

Many studies show that the joint design of precoding and selection achieves better performance of the network as compared to the decoupled design of precoding and selection [10], [14]. Joint design of user selection and precoding for multicell networks at the design level is addressed in [14], [15] where the user selection is introduced through binary variables which manipulate the rate or the precoding vector of the user. Moreover, a similar interpretation of scheduling binary variables arises in many other formulations [16], [17]. For example, in [17] the minimization of network cost in a cloud-radio access network scenario, the binary variable is multiplied with the precoding vector of BS. However, the aforementioned works dealing with joint design reduce the problem to one of precoding either by ignoring the Boolean variables [14] or by imposing sparsity constraints on precoding vectors [16], [17].

In this work, we propose a method to cast the joint design problem as a mixed integer non-linear programming (MINLP) that allows the joint update of user selection (binary) variables and precoding matrix without resorting to alternative update methods or relaxation methods. Further, we provide an iterative low complexity algorithm aiming at finding the stationary points of the NP-hard MINLP problem. To this end, we summarise below the contributions of this work:

- The user selection is equivalently handled through the norm of the precoding vector of the corresponding user, where non-zero power indicates the user being selected else considered not selected. Unlike the previous works, this step renders the optimization formulation amenable for joint design of user selection and precoding.

- The binary selection variables are relaxed, and the objective is penalized to promote a binary solution for the selection variables in the resulting relaxed non-convex joint design formulation. Further, a novel penalty function based on entropy that penalizes the selection variables as they shift away from 0 or 1 is considered.

- A novel reformulation of the rate which allows a refor- 
mulation of this non-convex joint design problem as a difference-of-concave (DC) functions thereby revealing the structure of WSR. Exploiting this, we propose a convex-concave procedure (CCP) based low complexity iterative algorithm to solve the DC problem, where a convex problem is solved in each iteration.

The rest of the paper is organised as follows. Section II presents the WSR maximization framework. Section III presents the joint problem formulation and its equivalent convex reformulations, followed by the proposed algorithm in Section IV. Section V presents simulation results, followed by conclusions in Section VI.

Notation: Lower or upper case letters represent scalars, lower case boldface letters represent vectors, and upper case boldface letters represent matrices. $\|\cdot\|$ represents the $\mathrm{Eu}-$ clidean norm, $|\cdot|$ represents the cardinality of a set or the magnitude of a scalar, $(\cdot)^{H},\left(\begin{array}{l}a \\ b\end{array}\right), \operatorname{tr}\{\}$ and $\mathbb{R}\{\}$ represent Hermitian transpose, $a$ choose $b$, trace and real operation.

\section{Weighted Sum Rate MaXimization}

We consider the multiuser MISO downlink system with $N$ single antenna users and a BS with $M(\leq N)$ antennas. BS transmits independent data to utmost $M$ users among $N$ users. This naturally leads to the selection of utmost $M$ users.

In this work, we consider the WSR maximization as the objective function. Towards formulating this, let $\mathcal{T}=\{1, \ldots N\}$ be the set containing indices of all users and $\mathcal{S}$ be the set containing all the possible subsets of $\mathcal{T}$ with cardinality less than or equal to $M$. The cardinality of $S$ is clearly $|\mathcal{S}|=\sum_{l=1}^{M}\left(\begin{array}{c}N \\ l\end{array}\right)$. Let $\mathbf{h}_{i} \in \mathbb{C}^{M \times 1}$ denote the downlink channel of user $i$ and $\mathbf{w}_{i} \in \mathbb{C}^{M \times 1}$ denote its corresponding precoding vector. Noise at each user is characterized to be additive white Gaussian with zero mean and variance $\sigma^{2}$. With the notations defined, the WSR maximization can be written as follows:

$$
\underbrace{\max _{\overline{\mathcal{S}} \in \mathcal{S}} \underbrace{\mathbf{W} \triangleq\left[\mathbf{w}_{1} \ldots, \mathbf{w}_{N}\right]}_{\text {precoding problem for scheduled users }} \sum_{(i) \subseteq \mathcal{\mathcal { S }}} \beta_{i} R_{i}}_{\text {Joint scheduer and Precoding problem }},
$$

where $\beta_{i}>0$ and $R_{i} \triangleq \log \left(1+\frac{\left|\mathbf{h}_{i}^{H} \mathbf{w}_{i}\right|^{2}}{\sigma^{2}+\sum_{j \neq i}^{N}\left|\mathbf{h}_{i}^{H} \mathbf{w}_{j}\right|^{2}}\right)$ are the weight and rate associated to the user $i$ respectively, and $\overline{\mathcal{S}}$ is the set containing indices of scheduled users. Weights $\beta_{i}$ s are assumed to be fixed according to some fairness criteria, and are given as input to the design. Different types of fairness metrics are proposed in the literature, e.g. fairness in terms of rates and allocated power are considered at the physical layer. We refer to [18] and references therein for details on fairness.

Design of user selection and precoding with the objective of maximizing WSR contains two coupled problems as shown in (1). The inner problem is the design of the precoding vector for the scheduled users with the objective of maximizing the WSR. The outer problem is the selection of users whose WSR is optimum among all other possible combinations. Obtaining a global solution to (1) requires an exhaustive search over two coupled spaces, i.e., selection and precoding. Exhaustive search algorithms, in general, have exponential complexity as the dimension of the problem increases. In the next section, we develop a method to formulate the problem (1) in a mathematically tractable way and propose a low-complexity suboptimal solution in the follow-up section.

\section{Joint User SELECTION AND PReCOding: Problem FORMULATION}

User selection is handled through the precoding vectors. The user $i$ is considered to be not selected if $\left\|\mathbf{w}_{i}\right\|_{2}=0$ and considered to selected otherwise. With the help of defined notations, the joint design problem can be mathematically formulated as

$$
\mathcal{P}_{1}: \max _{\mathbf{W}, \mathbf{P}, \boldsymbol{\eta}} \sum_{i=1}^{N} \beta_{i} R_{i}
$$

subject to $C_{1}: \quad \eta_{i} \in\{0,1\}, \forall i$,

$$
\begin{aligned}
C_{2}: & \left\|\mathbf{w}_{i}\right\|_{2}^{2} \leq P_{i} \eta_{i}, \forall i, \\
C_{3}: & \sum_{i=1}^{N} \eta_{i} \leq M, \\
C_{4}: & \sum_{i=1}^{N} P_{i} \leq P_{T},
\end{aligned}
$$

where $P_{i}$ and $\eta_{i}$ are the power and binary selection variables associate with user $i$, and $\mathbf{P}=\left[P_{1}, \ldots, P_{N}\right]^{T}$ and $\boldsymbol{\eta}=\left[\eta_{1}, \ldots, \eta_{N}\right]^{T}$.

Remarks:

- The problem $\mathcal{P}_{1}$ is non-convex with combinatorial constraints where the non-convexity is due to $\left\{R_{i}\right\}_{i=1}^{N}, \boldsymbol{\eta}$ and combinatorial nature is due to binary nature of $\boldsymbol{\eta}$. This is a mixed integer non-linear programming (MINLP) problem. Although efficient branch-and-bound methods exists for MINLP problem, it is not clear how these methods can be applied to $\mathcal{P}_{1}$.

- Binary nature of $\eta_{i}$ (i.e., $C_{1}$ ) together with constraint $C_{2}$ ensures user $i$ is either scheduled or not. In other words, $\eta_{i}=0$ leads to $\left\|\mathbf{w}_{i}\right\|_{2}^{2} \leq 0$ which leads to a precoding vector containing all zero entries, since norm is a nonnegative quantity. Similarly $\eta_{i}=1$ leads to $\left\|\mathbf{w}_{i}\right\|_{2}^{2} \leq P_{i}$.

- Constraint $C_{3}$ along with $C_{1}$ and $C_{2}$ guarantees that utmost $M$ users are scheduled.

Novelty of $\mathcal{P}_{1}$ lies in the formulation of scheduling constraint, $C_{2}$, and this reformulation is vital as it facilitates the joint update of $\boldsymbol{\eta}$ and $\mathbf{W}$ as discussed in the sequel. Note that this formulation differs from formulations in the literature ( [14], [15], [17] etc) where the scheduling constraint is handled by a binary slack variable which multiplies the precoding vector or the rate of the user, controlling the user scheduling. Thus, the multiplication not only make constraints non-convex but also make it difficult to obtain the joint update of Boolean and continuous variables due to the coupling of variables.

Since the problem $\mathcal{P}_{1}$ is a MINLP, it is difficult to achieve a global (or local) optimum for large dimensional systems 
in polynomial time. To circumvent the combinatorial nature of $\mathcal{P}_{2}$, the binary constraint $\eta_{i}$ is relaxed to a box constraint between 0 and 1 and $\eta_{i}$ is penalized so that the relaxed problem favours 0 or 1 . Letting $\mathbb{P}\left(\eta_{i}\right)$ to be the penalty function and $\lambda$ to be the penalty parameter, the resulting reformulation is written as,

$$
\begin{aligned}
\mathcal{P}_{2}: \max _{\mathbf{W}, \boldsymbol{\eta}} & \sum_{i=1}^{N}\left(\beta_{i} R_{i}+\lambda \mathbb{P}\left(\eta_{i}\right)\right) \\
\text { subject to } & C_{1}: 0 \leq \eta_{i} \leq 1, \forall i, \\
& C_{2}, C_{3}, \text { and } C_{4} \text { in }(2)
\end{aligned}
$$

We propose the penalty function defined as $\mathbb{P}\left(\eta_{i}\right) \triangleq$ $\eta_{i} \log \eta_{i}+\left(1-\eta_{i}\right) \log \left(1-\eta_{i}\right) \cdot \mathbb{P}\left(\eta_{i}\right)$ is a convex function in the feasible region $[0,1]$ and adds the least penalty at $\eta_{i}=0$ or 1 . This function induces huge penalty as $\eta_{i}$ s drifts away from 0 or 1 with the highest penalty at $\eta_{i}=0.5$. Hence, by choosing weighting parameter $\lambda$ appropriately, binary nature of $\eta_{i}$ can be enforced.

Now, the constraint set of $\mathcal{P}_{2}$ is convex since $C_{2}$ in $\mathcal{P}_{2}$ can be equivalently written as

$$
\left\|\left[\begin{array}{c}
2 \mathbf{w}_{i} \\
P_{i}-\eta_{i}
\end{array}\right]\right\|_{2} \leq P_{i}+\eta_{i}, \forall i .
$$

However, the problem $\mathcal{P}_{2}$ is still non-convex due to $\left\{R_{i}, \mathbb{P}\left(\eta_{i}\right)\right\}_{i=1}^{N} \mathrm{~s}$ in the objective. Towards obtaining an efficient algorithm addressing the problem above, $\mathcal{P}_{2}$ is equivalently reformulated with the help of a slack variable, $\gamma_{i}$, associated to user $i$, as,

$$
\mathcal{P}_{3}: \max _{\mathbf{W}, \mathbf{P}, \boldsymbol{\gamma}, \boldsymbol{\eta}} f(\boldsymbol{\gamma}, \boldsymbol{\eta}) \triangleq \sum_{i=1}^{N}\left(\beta_{i} \log \left(\gamma_{i}\right)+\lambda \mathbb{P}\left(\eta_{i}\right)\right)
$$

subject to $C_{1}, C_{2}, C_{3}, C_{4}$ in (3)

$$
\begin{aligned}
& C_{5}: \sigma^{2}+\sum_{j \neq i}^{N}\left|\mathbf{h}_{i}^{H} \mathbf{w}_{j}\right|^{2}-\frac{\sigma^{2}+\sum_{j=1}^{N}\left|\mathbf{h}_{i}^{H} \mathbf{w}_{j}\right|^{2}}{\gamma_{i}} \leq 0, \forall i \\
& C_{6}: \gamma_{i} \geq 1 ; \forall i,
\end{aligned}
$$

where $\gamma=\left[\gamma_{1}, \ldots, \gamma_{N}\right]^{T}$, and $C_{5}$ in $\mathcal{P}_{3}$ is the rearrangement of $\gamma_{i} \leq\left(1+\frac{\left|\mathbf{h}_{i}^{H} \mathbf{w}_{i}\right|^{2}}{\sigma^{2}+\sum_{j \neq i}^{N}\left|\mathbf{h}_{i}^{H} \mathbf{w}_{j}\right|^{2}}\right)$.

Now, notice that the novel reformulation of $R_{i}$ makes the objective in $\mathcal{P}_{3}$ a difference of two concave functions i.e. $f(\boldsymbol{\gamma}, \boldsymbol{\eta})=\sum_{i=1}^{N}\left(\beta_{i} \log \left(\gamma_{i}\right)\right)-\left(-\sum_{i=1}^{N} \lambda \mathbb{P}\left(\eta_{i}\right)\right)$ and the constraint $C_{5}$ is a difference of two convex functions. Hence, the problem $\mathcal{P}_{3}$ belongs to the class of DC programming. The semidefinite relaxation version of WSR maximization problem is formulated as a DC program in many previous works, [19], [20], by translating the WSR maximization problem into a semidefinite relaxed problem. However, to the best of our knowledge, no prior work exists which translates the WSR maximization of the form in $\mathcal{P}_{3}$ as a DC problem.

\section{Joint User Selection And Precoding: Design ALGORITHM}

In this section, we propose a convex-concave procedure (CCP) [21] based iterative algorithm to the DC problem in
(4). CCP is a powerful tool to find a stationary point of DC programming problems. Within this framework, an iterative procedure is performed, wherein the two steps of Convexification and Optimization are executed in each iteration. The convexification step lower bounds the cost function with a surrogate concave function where the lower bound is tight at the previous iteration. The optimization step then solves the surrogate convex subproblem globally. Thus, the proposed joint selection and precoding (JSP) algorithm iteratively executes the following two steps until the convergence:

- Convexification: Let $\mathbf{W}^{k-1}, \boldsymbol{\eta}^{k-1}, \boldsymbol{\gamma}^{k-1}$ be the estimates of $\mathbf{W}, \boldsymbol{\eta}, \boldsymbol{\gamma}$ in iteration $k-1$ and $g_{i}\left(\mathbf{W}, \gamma_{i}\right)=$ $\frac{\sigma^{2}+\sum_{j=1}^{N}\left|\mathbf{h}_{i}^{H} \mathbf{w}_{j}\right|^{2}}{\gamma_{i}}$. In iteration $k$, the convex part of the objective, $\left(-\sum_{i=1}^{N} \lambda P\left(\eta_{i}\right)\right)$, and the concave part of constraint are replaced by their affine approximations around the estimate of $\left(\mathbf{W}^{k-1}, \boldsymbol{\eta}^{k-1}, \boldsymbol{\gamma}^{k-1}\right)$

- Optimization: Update $\left(\mathbf{W}^{k+1}, \mathbf{P}^{k+1}, \boldsymbol{\eta}^{k+1}, \boldsymbol{\gamma}^{k+1},\right)$ is obtained by solving the following convex problem:

$$
\begin{gathered}
\mathcal{P}_{4}: \max _{\mathbf{W}, \mathbf{P}, \boldsymbol{\gamma}, \boldsymbol{\eta}} \sum_{i=1}^{N}\left(\beta_{i} \log \left(\gamma_{i}\right)+\lambda \eta_{i} \nabla \mathbb{P}\left(\eta_{i}^{k-1}\right)\right) \\
\text { subject to } C_{1}, C_{2}, C_{3}, C_{4} \text { and } C_{6} \text { in (4) } \\
C_{5}: \sigma^{2}+\sum_{j \neq i}^{N}\left|\mathbf{h}_{i}^{H} \mathbf{w}_{j}\right|^{2}-g_{i}\left(\mathbf{W}^{k-1}, \gamma_{i}^{k-1}\right)- \\
\mathbb{R}\left\{\operatorname{tr}\left\{\left(\nabla^{H} g_{i}\left(\mathbf{W}^{k-1}, \gamma_{i}^{k-1}\right)\left[\begin{array}{c}
\mathbf{W}-\mathbf{W}^{k-1} \\
\gamma_{i}-\gamma_{i}^{k-1}
\end{array}\right]\right)\right\}\right\} \leq 0, \forall i
\end{gathered}
$$

Note that the proposed JSP algorithm is based on CCP framework. It is well known that a feasible initial point is sufficient for the CCP procedure to converge to a stationary point (kindly refer [22]). One can find an initial feasible point heuristically and the solution proposed in [5] is a valid feasible initial $\mathbf{W}$ and a feasible $\eta$ is chosen by making entries of corresponding scheduled users to 1 .

JSP is an iterative algorithm, and hence, the complexity of the algorithm depends on the convex sub-problem $\mathcal{P}_{4}$. The problem $\mathcal{P}_{4}$ is a convex problem and commercial software such as CVX can solve the problem efficiently even in large dimensions. Through simulations, we observe that JSP typically converges to a stationary point in 10-15 iterations. Thus, the proposed algorithm is computationally efficient, and as we show in the next section, superior in performance compared to the state-of-the-art algorithms.

\section{Simulation RESUlTS}

In this section, we evaluate the performance of the proposed JSP. In the benchmark algorithms use for comparison, the users are scheduled according to the SUS approach proposed in [5], followed by ZF precoder, referred to as SUS-ZF, and MMSE precoder referred as SUS-MMSE. Further, the total power, $P_{T}$, is optimally allocated to the SUS-MMSE and SUS-ZF users using the water-filling algorithm. The system parameters defined below are common for all the plots. Entries 
of the channel matrix, i.e., $\left\{h_{i j}\right\}$ are drawn from the complex normal distribution with zero mean and unit variance. All user channels $\mathbf{h}_{i}$ are normalized to unit power. User weights and noise variances are considered to be unity i.e., $\beta_{i}=\sigma^{2}=1$, $\forall i$ and $\lambda$ is initialized to 0 and incremented in steps of 0.1 . For SUS, $\alpha$, which is defined as a measure of semi-orthogonality of users in [5], is chosen optimally for each combination of $M$ and $N$. The precoding vectors of SUS-ZF, as given in $\mathcal{P}_{4}$, are used as the initial feasible solution to JSP.

In figure 1, the WSR performance of JSP and the benchmark schemes are plotted as a function of $N$ for $P_{T}=10 \mathrm{~dB}$ for two following system configurations: $M=4$ in Figure (1a) and $M=8$ in Figure (1b). It can be clearly seen from these plots that by jointly designing selection and precoding, JSP outperforms SUS-ZF and SUS-MMSE. In the right most region of Figure (1a), i.e. $N$ in the range $40-50$, JSP outperforms only by a small margin, since SUS can find non-interfering users with high probability as $N \rightarrow 50$. However, for $N$ in the range of $10-30$, both the benchmark algorithms are outperformed by JSP by large margin in Figure (1a) and Figure (1b). Since, the SUS fails to find near orthogonal users as $M \rightarrow N$, ZF and MMSE precoders spend the available power in canceling the interference. However, due to the systematic joint design, JSP outperforms both the benchmarks by a larger margin in Figure (1a) and (1b), as $M \rightarrow N$ i.e for $N$ in the range of 10-30. The relatively higher performance gap in Figure (1b) for $N$ in the range 10 to 30 is due to the selection of relatively higher number of users i.e. $M=8$.

In figure 2, we plot the rates of the users as a function of the iteration index for one channel realization of the system with $M=8, N=30$. In Figure (2a), SUS-ZF is performed with the optimal value of $\alpha=0.27$ achieving the sum rate (SR) of $5.97 \mathrm{bps} / \mathrm{Hz}$. In Figure (2b), $\alpha$ is tuned to 0.4 achieving SR of $4.9932 \mathrm{bps} / \mathrm{Hz}$, for the same number of scheduled number users as JSP (7 here). The yellow line at iteration 1 in figure ( 2a) and (2b), indicates the scheduled users of SUS-ZF. In both of the plots, JSP is initialized with the solution of SUS-ZF with $\alpha=0.27$. Due to the systematic joint design, over the iterations, JSP explores other combinations of selection and precoding which achieves the better TSE of $7.79 \mathrm{bps} / \mathrm{Hz}$.

\section{Vi. Conclusions}

In this paper, the joint selection and precoding problem was considered for a multiuser MISO downlink channel. Unlike the existing works, the design is formulated to solve the problem jointly. Noticing that the original problem to be MINLP problem, we have developed an efficient formulation to relax the problem to a structured DC programming problem. Finally, we proposed an efficient low-complexity CCP based iterative algorithm to solve DC problem, which is guaranteed to converge to a stationary point. Through simulations, we established the attractiveness of the proposed one-stage joint techniques with respect to the two-stage benchmark solutions.

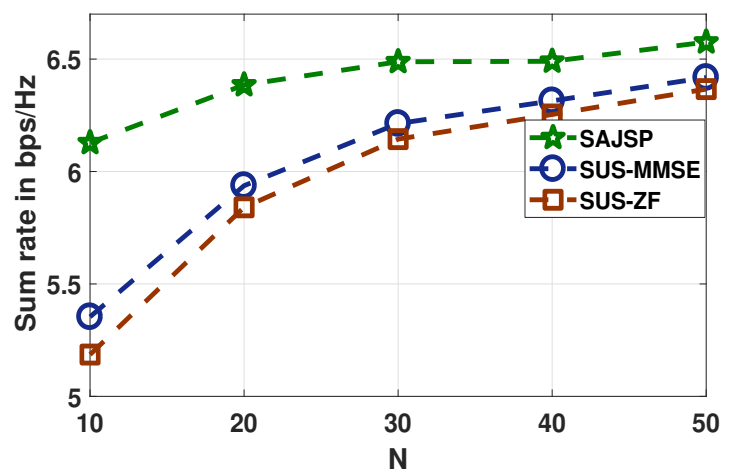

(a) $M=4$ and $N$ in the range from 10 to 50 insteps of 10

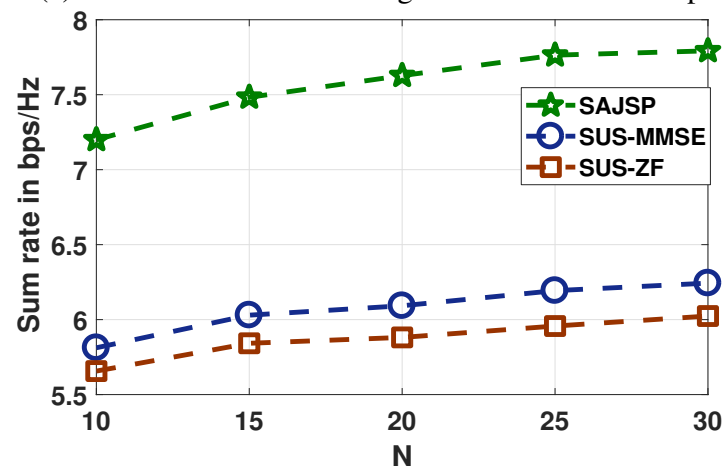

(b) $M=8$ and $N$ in the range from 10 to 30 insteps of 5 .

Fig. 1: WSR versus $N$ of a single cell MISO system for $P_{T}=10 \mathrm{~dB}$.

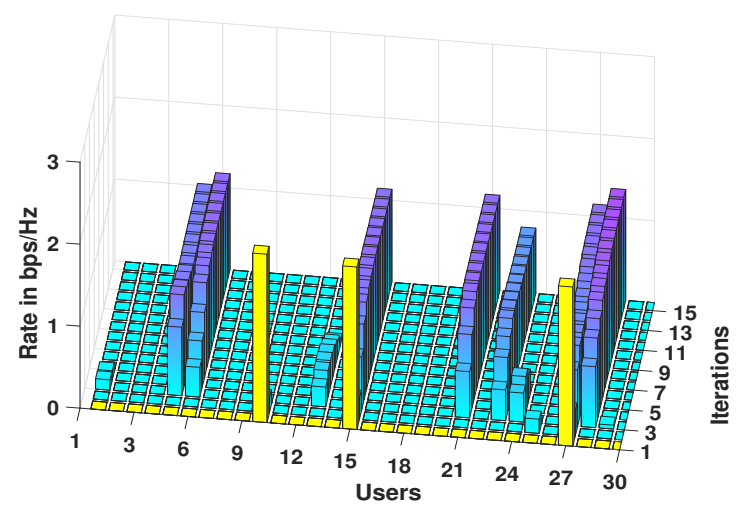

(a) SUS-ZF with 3 scheduled users.

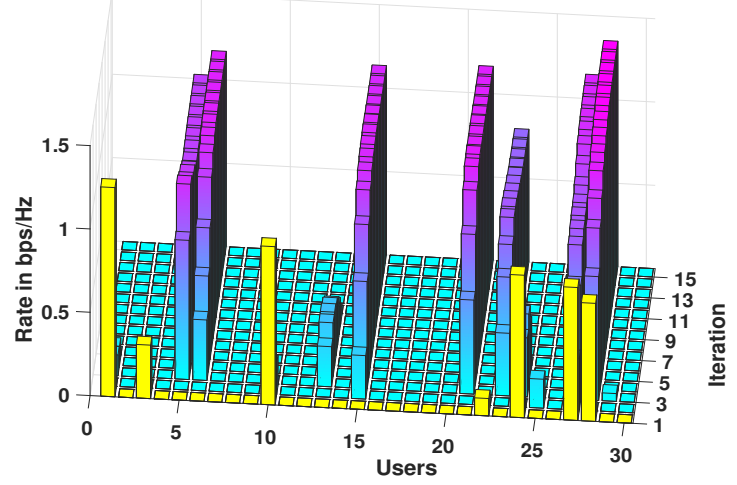

(b) SUS-ZF with 7 scheduled users.

Fig. 2: Rates evolution of users for the following system parameters: $M=$ $8, N=30, P_{T}=10 \mathrm{~dB}$ in a single cell MISO system. 


\section{REFERENCES}

[1] H. Viswanathan, S. Venkatesan, and H. Huang, "Downlink capacity evaluation of cellular networks with known-interference cancellation," IEEE J. Sel. Areas Commun., vol. 21, no. 5, pp. 802-811, June 2003.

[2] P. Zetterberg and B. Ottersten, "The spectrum efficiency of a base station antenna array system for spatially selective transmission," IEEE $J^{\cdot} V T$, vol. 44, no. 3, pp. 651-660, Aug 1995.

[3] S. Anderson, M. Millnert, M. Viberg, and B. Wahlberg, "An adaptive array for mobile communication systems," IEEE J'VT, vol. 40, no. 1 , pp. 230-236, Feb 1991.

[4] G. Dimic and N. D. Sidiropoulos, "On downlink beamforming with greedy user selection: performance analysis and a simple new algorithm," IEEE Trans. Signal Process., vol. 53, no. 10, pp. 3857-3868, Oct 2005.

[5] T. Yoo and A. Goldsmith, "On the optimality of multiantenna broadcast scheduling using zero-forcing beamforming," IEEE J. Sel. Areas Commun., vol. 24, no. 3, pp. 528-541, March 2006.

[6] Z. Q. Luo and S. Zhang, "Dynamic spectrum management: Complexity and duality," IEEE Journal of Selected Topics in Signal Processing, vol. 2, no. 1, pp. 57-73, Feb 2008.

[7] Y. F. Liu, Y. H. Dai, and Z. Q. Luo, "Coordinated beamforming for miso interference channel: Complexity analysis and efficient algorithms," IEEE Trans. Signal Process., vol. 59, no. 3, pp. 1142-1157, March 2011.

[8] D. Christopoulos, S. Chatzinotas, and B. Ottersten, "Multicast multigroup precoding and user scheduling for frame-based satellite communications," IEEE Trans. Wireless Commun., vol. 14, no. 9, pp. 4695-4707, Sept 2015.

[9] K. Hamdi, W. Zhang, and K. B. Letaief, "Opportunistic spectrum sharing in cognitive mimo wireless networks," IEEE Trans. Wireless Commun., vol. 8, no. 8, pp. 4098-4109, August 2009.

[10] W. Yu, T. Kwon, and C. Shin, "Multicell coordination via joint scheduling, beamforming, and power spectrum adaptation," IEEE Trans. Wireless Commun., vol. 12, no. 7, pp. 1-14, July 2013.

[11] S. H. Moon, C. Lee, S. R. Lee, and I. Lee, "Joint user scheduling and adaptive intercell interference cancelation for miso downlink cellular systems," IEEE Trans. Veh. Technol., vol. 62, no. 1, pp. 172-181, Jan 2013.

[12] M. Li, I. B. Collings, S. V. Hanly, C. Liu, and P. Whiting, "Multicell coordinated scheduling with multiuser zero-forcing beamforming," IEEE Trans. Wireless Commun., vol. 15, no. 2, pp. 827-842, Feb 2016.

[13] X. Huang, G. Xue, R. Yu, and S. Leng, "Joint scheduling and beamforming coordination in cloud radio access networks with qos guarantees," IEEE Trans. Veh. Technol., vol. 65, no. 7, pp. 5449-5460, July 2016.

[14] M. L. Ku, L. C. Wang, and Y. L. Liu, "Joint antenna beamforming, multiuser scheduling, and power allocation for hierarchical cellular systems," IEEE J. Sel. Areas Commun., vol. 33, no. 5, pp. 896-909, May 2015.

[15] A. Douik, H. Dahrouj, T. Y. Al-Naffouri, and M. S. Alouini, "Coordinated scheduling and power control in cloud-radio access networks," IEEE Transactions on Wireless Communications, vol. 15, no. 4, pp. 2523-2536, April 2016.

[16] B. Dai and W. Yu, "Sparse beamforming and user-centric clustering for downlink cloud radio access network," IEEE Access, vol. 2, pp. 13261339, 2014.

[17] M. Tao, E. Chen, H. Zhou, and W. Yu, "Content-centric sparse multicast beamforming for cache-enabled cloud ran," IEEE Trans. Wireless Commun., vol. 15, no. 9, pp. 6118-6131, Sept 2016.

[18] H. SHI, R. V. Prasad, E. Onur, and I. G. M. M. Niemegeers, "Fairness in wireless networks:issues, measures and challenges," IEEE Commun. Surveys Tuts., vol. 16, no. 1, pp. 5-24, First 2014.

[19] S. He, J. Wang, Y. Huang, B. Ottersten, and W. Hong, "Codebook-based hybrid precoding for millimeter wave multiuser systems," IEEE $J \cdot S P$, vol. 65 , no. 20, pp. 5289-5304, Oct 2017.

[20] C. T. K. Ng and H. Huang, "Linear precoding in cooperative mimo cellular networks with limited coordination clusters," IEEE Journal on Selected Areas in Communications, vol. 28, no. 9, pp. 1446-1454, December 2010.

[21] A. L. Yuille and A. Rangarajan, "The concave-convex procedure (CCCP)," in NIPS, 2001.

[22] G. R. Lanckriet and B. K. Sriperumbudur, "On the convergence of the concave-convex procedure," in Advances in Neural Information Processing Systems 22, 2009, pp. 1759-1767. 